\title{
Article
}

\section{Templated Quasicrystalline Molecular Ordering}

Smerdon, Joe, Young, K. M, Lowe, M., Hars, S. S., Yadav, T. P., Hesp, D., Dhanak, V. R., Tsai, A. P., Sharma, H. R. and McGrath, R.

Available at https://clok.uclan.ac.uk/10512/

Smerdon, Joe orcid iconORCID: 0000-0002-7387-8362, Young, K. M, Lowe, M., Hars, S. S., Yadav, T. P., Hesp, D., Dhanak, V. R., Tsai, A. P., Sharma, H. R. et al (2014) Templated Quasicrystalline Molecular Ordering. Nano Letters, 14 (3). pp. 1184-1189. ISSN 1530-6984

It is advisable to refer to the publisher's version if you intend to cite from the work. http://dx.doi.org/10.1021/nl403947b

For more information about UCLan's research in this area go to http://www.uclan.ac.uk/researchgroups/ and search for <name of research Group>.

For information about Research generally at UCLan please go to http://www.uclan.ac.uk/research/

All outputs in CLoK are protected by Intellectual Property Rights law, including Copyright law. Copyright, IPR and Moral Rights for the works on this site are retained by the individual authors and/or other copyright owners. Terms and conditions for use of this material are defined in the policies page.

\section{CLoK}

Central Lancashire online Knowledge www.clok.uclan.ac.uk

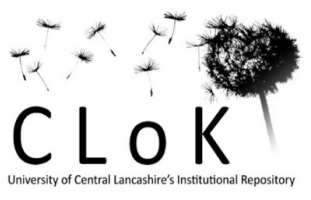




\title{
Templated quasicrystalline molecular ordering
}

\author{
J. A. Smerdon ${ }^{1},{ }^{* \dagger}$ K. M. Young ${ }^{2},{ }^{\dagger}$ M. Lowe ${ }^{2}{ }^{\dagger}$ S. S. $\operatorname{Hars}^{2}{ }^{\dagger}{ }^{\dagger}$ T. P. Yadav $^{2}{ }^{\dagger}{ }^{\text {D. D. }}$ \\ Hesp $^{2},{ }^{\dagger}$ V. R. Dhanak ${ }^{2}{ }^{\dagger}$ A. P. Tsai ${ }^{3},{ }^{\dagger}$ H. R. Sharma ${ }^{2}{ }^{\dagger}$ and R. McGrath ${ }^{2 \dagger}$ \\ ${ }^{1}$ Jeremiah Horrocks Institute for Mathematics, Physics and Astronomy, University of \\ Central Lancashire, Preston, PR1 2HE, UK, ${ }^{2}$ Department of Physics, University of \\ Liverpool, Oxford Street, L69 7ZE, UK, and ${ }^{3}$ Institute of Multidisciplinary Research for \\ Advanced Materials, Tohoku University, Sendai, 980-8577, Japan \\ E-mail: jsmerdon@uclan.ac.uk
}

\begin{abstract}
Quasicrystals are materials with long range ordering but no periodicity. We report scanning tunneling microscopy (STM) observations of quasicrystalline molecular layers on five-fold quasicrystal surfaces. The molecules adopt positions and orientations on the surface consistent with the quasicrystalline ordering of the substrate. Carbon-60 adsorbs atop sufficiently-separated $\mathrm{Fe}$ atoms on icosahedral $\mathrm{Al}-\mathrm{Cu}-\mathrm{Fe}$ to form a unique quasicrystalline lattice whereas further $\mathrm{C}_{60}$ molecules decorate remaining surface $\mathrm{Fe}$ atoms in a quasi-degenerate fashion. Pentacene (Pn) adsorbs at tenfold-symmetric points around surface-bisected rhombic triacontahedral clusters in icosahedral Ag-In$\mathrm{Yb}$. These systems constitute the first demonstrations of quasicrystalline molecular ordering on a template.
\end{abstract}

\footnotetext{
${ }^{*}$ To whom correspondence should be addressed

${ }^{\dagger 1}$ Jeremiah Horrocks Institute for Mathematics, Physics and Astronomy, University of Central Lancashire, Preston, PR1 2HE, UK

${ }^{\ddagger 2}$ Department of Physics, University of Liverpool, Oxford Street, L69 7ZE, UK

${ }^{\top 3}$ Institute of Multidisciplinary Research for Advanced Materials, Tohoku University, Sendai, 980-8577, Japan
} 
Keywords: molecules, quasicrystal, scanning tunneling microscopy, pentacene, fullerene

Quasicrystals, discovered by Dan Shechtman in 1982 and published in $1984,{ }^{1}$ changed the face of crystallography and condensed matter physics. The discovery of alloys with icosahedral ordering gave new meaning to the examination of aperiodic ordering, previously a mathematical recreation, ${ }^{2}$ and now condensed matter literature records hundreds of quasicrystalline phases in mostly ternary alloys, though at least one family of binary quasicrystals exists. ${ }^{3}$ Quasicrystalline alloys have high hardness, low surface energy and non-stick properties comparable to Teflon. The use of these materials as templates has led to the discovery of several quasicrystalline single-element systems. ${ }^{4-12}$

Recently, most interest in quasicrystals is due to the generalization of aperiodic ordering to several classes of systems. Soft matter quasicrystals exhibiting dodecagonal symmetries have been reported. ${ }^{13}$ Dodecagonal ordering is also observed in extended two-dimensional arrays of nanoparticles. ${ }^{14}$ Colloids have been induced by laser interference patterns to form quasicrystalline networks. ${ }^{15}$ The growth of quasicrystalline perovskite thin films has also recently been reported. ${ }^{16}$

In periodic materials, the highest degree of symmetry is provided by the cubic system, which is isotropic in six directions. There is no upper limit on the degree of symmetry in quasicrystalline systems. In 2-d, 12-fold symmetry is observed in several classes of system. ${ }^{13,14}$ Icosahedral ordering is the only 3 -d aperiodic ordering observed in hierarchical self-assembled systems. ${ }^{1}$ Compared to periodic materials, these provide a closer approximation to an isotropic first Brillouin zone, which is of great importance to the transmission of waves through quasicrystalline lattices, and has led to the design of photonic metamaterials which exhibit negative refractive indices and near-isotropic band gaps. ${ }^{17}$

One 'patch' of quasicrystalline material is unlikely to be identical to any other similarly sized patch, with the number of permutations increasing exponentially with the size of the patch. This has led to parallels drawn between quasicrystals and molecular adsorption systems such as the 'random tilings' described by Blunt et al., of rhombic 
molecules with a completely defined lattice but with orientation unconstrained except by neighboring molecules. ${ }^{18}$

If molecules could be induced to order in a quasicrystalline fashion, then this would open the possibility of manufacturing molecular materials with tuned optical properties, or of producing quasiperiodic magnetic systems through ordering of magnetic molecules leading to the possibility of exploring frustrated quasicrystalline magnetic systems, such as that demonstrated by Vedmedenko et al., on the molecular scale. ${ }^{19}$ Up to now, previous attempts have been unsuccessful. ${ }^{20-23}$ In this work we demonstrate two examples of templated growth of quasicrystalline molecular layers. $\mathrm{C}_{60}$ on face-centered icosahedral $\mathrm{Al}-\mathrm{Cu}-\mathrm{Fe}$ and $\mathrm{Pn}$ on simple icosahedral Ag-In-Yb form extended quasicrystalline networks with well-defined nucleation sites. The rod-shaped Pn molecules exhibit ordering through their orientations as well as their locations.

Several depositions of $\mathrm{C}_{60}$ on $\mathrm{Al}-\mathrm{Cu}-\mathrm{Fe}$ were conducted, with the substrate temperature during deposition ranging from $773 \mathrm{~K}$ to $973 \mathrm{~K}$. At $973 \mathrm{~K}$ a clean Al-Cu-Fe surface with patches of disordered material was recovered. Figure 1 shows $\mathrm{C}_{60}$ on $\mathrm{Al}$ $\mathrm{Cu}-\mathrm{Fe}$ deposited with the substrate at $773 \mathrm{~K}$. The face-centered icosahedral Al-Cu-Fe quasicrystal, based on pseudo-Mackay icosahedra (pMI), is isostructural to icosahedral $\mathrm{Al}-\mathrm{Pd}-\mathrm{Mn},{ }^{24,25}$ as is the annealed surface. ${ }^{26}$ The $\mathrm{Al}$ network is identical, and $\mathrm{Cu}$ and Fe atoms occupy the $\mathrm{Pd}$ and Mn positions respectively.

'Dark stars' - large surface vacancies - have previously inspired investigation into Al-Pd-Mn as a substrate for molecular adsorption. ${ }^{27}$ Some $\mathrm{C}_{60}$ molecules arrange in quasiperiodically located sites, but far more molecules are randomly located. ${ }^{20}$ For $\mathrm{C}_{60} / \mathrm{Al}-\mathrm{Cu}-\mathrm{Fe}$, shown in Figure 1(a) and in detail in Figure 1(b), all molecules occupy quasiperiodic sites. The molecules can be split into two categories: those which appear bright $(B)$ and those which appear $\operatorname{dim}(D)$. The tenfold symmetry of the $B$-molecules is clearly visualized in the autocorrelation of molecule locations in Figure 1(d). STM images comparing the substrate to the model are shown in Supplemental Information Figure S1. To ensure that the autocorrelation maps reflect solely the ordering of the molecules, they are generated from molecular positions extracted from the STM images, 

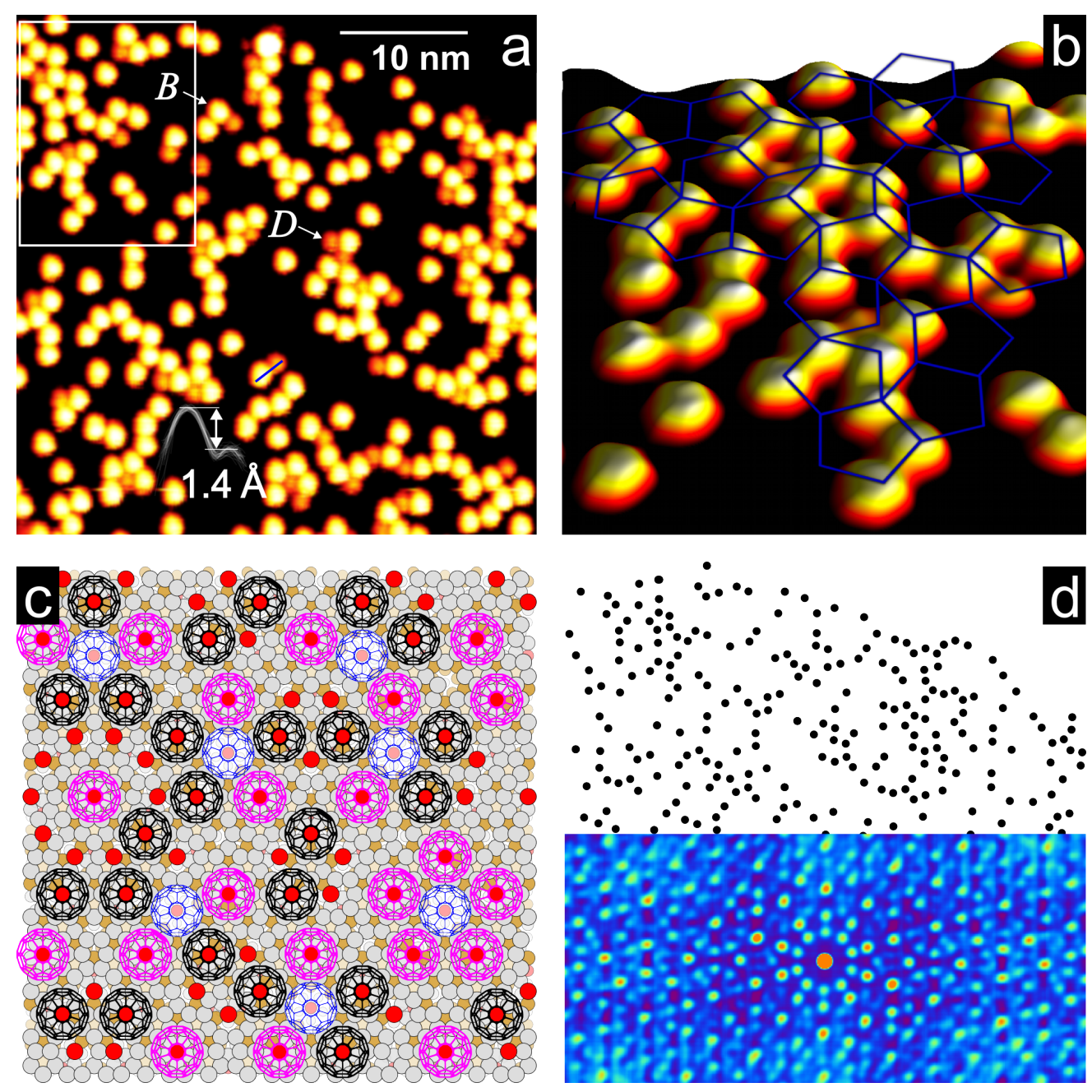

Figure 1: Quasicrystalline $C_{60}$ layer on icosahedral Al-Cu-Fe.(a) $50 \mathrm{~nm} \times 50 \mathrm{~nm}$ STM image of $\mathrm{C}_{60}$ molecules decorating the Al-Cu-Fe surface $\left(V_{\text {gap }}=-1.3 \mathrm{~V}, I_{T}=0.4 \mathrm{nA}\right)$. The molecules indicated are examples of the $B$ and $D$ molecules described in the text. (b) The detail of (a) indicated with a patch of a Penrose P1 tiling superimposed. (c) A saturated quasicrystalline lattice of $\mathrm{C}_{60}$ atop a $10 \mathrm{~nm}$ square patch of $\mathrm{Al}-\mathrm{Cu}-\mathrm{Fe}$ ( $\mathrm{Al}$ grey, $\mathrm{Cu}$ brown, Fe red). Magenta molecules decorate a unique lattice, as there are no nearby Fe atoms to provide competing alternative adsorption sites. Blue molecules decorate subsurface pseudoMackay icosahedra (pMI) centers. (d) The positions of the bright molecules extracted with the associated autocorrelation pattern, showing tenfold symmetry of the layer. The fast scan direction $x$ shows excellent ordering, but the slow scan direction $y$ shows some decay due to drift.

rather than the STM images themselves. Further datasets are shown in Supplemental Information Figures S2 and S3, along with a detailed description of the data analysis procedure employed.

A fundamental measurement of a quasicrystalline layer is the nearest neighbor dis- 
tance, which is also the smallest edge length $a$ in a pentagonal tiling connecting constituents of the layer. Larger tilings that also connect constituents of the layer can be expressed in the form $\tau^{n} \times a$, where $\tau(=1.618 \ldots)$ is the golden ratio.

At this sub-saturation coverage, the $B$ molecules sparsely occupy a pentagonal lattice with $a=1.2 \pm 0.1 \mathrm{~nm}$. This characteristic separation is consistent with an adsorption site dependent on surface Fe atoms. There are no $\mathrm{Cu}$ atoms in the top layer of the substrate, and the $\mathrm{Al}$ atoms are densely packed. The Fe atoms, while too close together for all to be decorated $\left(a_{F e}=a \times \tau^{-1}=0.74 \mathrm{~nm}, \mathrm{C}_{60}\right.$ van der Waals diameter $=1.0 \mathrm{~nm}$ ) provide a sparse nucleation site network which can explain all of the observed features of the $B$ molecule lattice. We revisit this later in the discussion.

Despite ambiguities in quasicrystal models, the observation that the nucleation site network for an ordered adlayer depends on the distribution of the least abundant constituent element is reasonably well-supported for all five-fold quasicrystalline adlayers discovered thus far. For Pb/A-lPb-Mn, ${ }^{6} \mathrm{Si} / \mathrm{Al}-\mathrm{Pd}-\mathrm{Mn}^{8}$ and $\mathrm{Bi} / \mathrm{Al}-\mathrm{Pd}-\mathrm{Mn},{ }^{5}$ the network depends on Mn atoms (at the centers of 'white flowers') in the surface layer. For $\mathrm{C}_{60} / \mathrm{Al}-\mathrm{Pd}-\mathrm{Mn}$ and $\mathrm{Ag} / \mathrm{Al}-\mathrm{Pd}-\mathrm{Mn},{ }^{28}$ those molecules in ordered sites decorate the dark stars in the substrate, which occur atop $\mathrm{Mn}$ atoms in the subsurface layer. ${ }^{20} \mathrm{For} \mathrm{Al} / \mathrm{Al}-$ $\mathrm{Cu}-\mathrm{Fe}$, the network is argued to be analogous to the dark star network in isostructural Al-Pd-Mn, based on subsurface Mn atoms, which leads to the conclusion that, in Al$\mathrm{Cu}-\mathrm{Fe}$, it is based on $\mathrm{Fe}$ atoms. ${ }^{9}$ For a detailed review of adsorption sites, we refer the reader to Reference $29 .^{29}$

The $D$ molecules occupy a different lattice to the $B$ molecules, with $a_{D}=\tau \times a$. The molecules often appear at the centers of incomplete pentagonal clusters of $B$ molecules and their height is around $0.14 \mathrm{~nm}$ lower than the $B$ molecules. The locations of these molecules fits well with the expected locations of Fe atoms in the next layer from the model and, importantly, not with any Fe atoms in the surface layer of the model. These observations support the idea that $D$ molecules are adsorbed atop sub-surface Fe atoms, and further support the basic thesis that all of the molecular locations are dependent on the network of $\mathrm{Fe}$ atoms in the uppermost layers of the quasicrystal. Both $\mathrm{Cu}$ and 
$\mathrm{Al}$ atoms are too densely packed - thus providing a multiplicity of potential conflicting nucleation sites - to influence the molecular ordering. For subsurface Fe atoms to be decorated, some $\mathrm{Al}$ atoms must relocate. We thus conclude that the presence of $\mathrm{C}_{60}$ at high temperature activates a moderate surface reconstruction based on the affinity between $\mathrm{C}_{60}$ and subsurface Fe atoms. The separation in the quasicrystal bulk of Fe layers is $0.21 \mathrm{~nm}$, significantly greater than the height difference observed between $B$ and $D$ molecules. This could be due to a relaxation of the surface layers of the quasicrystal, although a dynamical LEED investigation of the clean surface found that such a relaxation served to separate the upper Fe-containing layers rather than bring them together. ${ }^{30}$ This phenomenon is therefore more likely due to an electronic effect or to the different adsorption environment of $D$ molecules compared to $B$ molecules.

No intramolecular resolution was obtained during this experiment. Although it is possible that the $\mathrm{C}_{60}$ molecules are rotationally disordered, it seems reasonable to expect that they adsorb with a pentagonal facet parallel to the surface to maximise coordination with substrate atoms.

An interesting feature of this film is the coexistence of several exclusive competing adsorption sites - quasi-degeneracy, defined as the existence of many states of similar energy. On periodic substrates, molecules diffuse to produce long-range ordered layers, stick where they land to produce disordered layers, or undergo some other interaction, for example, the formation of a sparse layer based on dipole repulsion. Molecules which can adopt several relative orientations with comparable energies produce quasidegenerate layers with well-defined lattices such as the 'random tiling' reported by Blunt et al. ${ }^{18} \mathrm{Pn}$ on $\mathrm{Cu}(111)$ forms a random tiling with well defined orientations but a random lattice. ${ }^{31}$ However, $\mathrm{C}_{60} / \mathrm{Al}-\mathrm{Cu}-\mathrm{Fe}$ is the first system to be observed in which molecules only adsorb on one subset of points on a unique lattice. This is due to the size of the substrate lattice of adsorption sites, which has a characteristic distance $a$ large enough that it can directly affect molecular adsorption and small enough that not all sites can be decorated. The $10 \mathrm{~nm} \times 10 \mathrm{~nm}$ patch of the model of $\mathrm{C}_{60} / \mathrm{Al}-\mathrm{Cu}-\mathrm{Fe}$ shown can accommodate approximately $5^{5} \times 4^{5} \times 3^{3} \times 2^{3} \approx 10^{9}$ distinct arrangements, 
if we consider that each pentagonal arrangement of edge length $\mathrm{a}=1.2 \mathrm{~nm}$ can support as many arrangements as it has members (i.e., a full pentagon can support 5 distinct arrangements, a pentagonal feature of only 4 atoms can support 4 distinct arrangements etc). The figure is approximate because we consider that the incomplete pentagons around the large decagonal feature towards the left of the model do not interact, leading to a degree of overestimation.

Saturated layers additionally have a unique non-degenerate lattice where $a^{\prime}=a_{D}=$ $\tau \times a=1.9(4) \mathrm{nm}$, in which the sites are sufficiently separated that they can all be decorated, as they have no nearby competing sites. These molecules are colored magenta in Figure 1(c), with many atop the centers of surface-bisected pMIs. A pMI in Al-Cu-Fe has an Fe atom at the center surrounded by an $\mathrm{Al}$ core, a dodecahedron consisting of $\mathrm{Al} / \mathrm{Cu}$ or $\mathrm{Mn} / \mathrm{Cu}$ and an outer icosidodecahedron consisting of $\mathrm{Cu} / \mathrm{Al}$ or $\mathrm{Al}$ only. The sites with $a=1.2 \mathrm{~nm}$ form the quasi-degenerate lattice described above. The lattice of the next layer down of $\mathrm{Fe}$ is inverted compared to that of surface Fe. The mechanism that selects which Fe atoms are exposed by the high-temperature $\mathrm{C}_{60}$ induced reconstruction might be based on whether the Fe atoms are at the centers of pMI: these Fe atoms have only $\mathrm{Al}$ atoms as nearest neighbors and also a separation consistent with our data. As mentioned above, the notion of a nucleation site network partially based on pMI centers is consistent with reports of atomic nucleation on isostructural Al-Pd-Mn. ${ }^{5,8}$

In a separate experiment, Pn was adsorbed at room temperature on an $A_{42} \operatorname{In}_{42} \mathrm{Yb}_{16}$ quasicrystal, an icosahedral quasicrystal isostructural to the binary quasicrystal Cd-Yb. Within this model, we cannot differentiate between Ag and In atoms. The surface of $\mathrm{Ag}-\mathrm{In}-\mathrm{Yb}$ is composed of high density planes which bisect the rhombic triacontahedral (RTH) clusters that make up the quasicrystalline bulk structure. ${ }^{32} \mathrm{Pn}$ is a rod-shaped molecule consisting of five acene rings fused along C-C bonds. Previous observations of monolayer Pn on icosahedral Al-Pd-Mn and various metallic substrates find no intramolecular resolution. ${ }^{22,31,33,34}$ Resolution of molecular orbitals is obtained when the Pn molecule is adsorbed on some decoupling film, ${ }^{31,35}$ under which conditions the seven- 

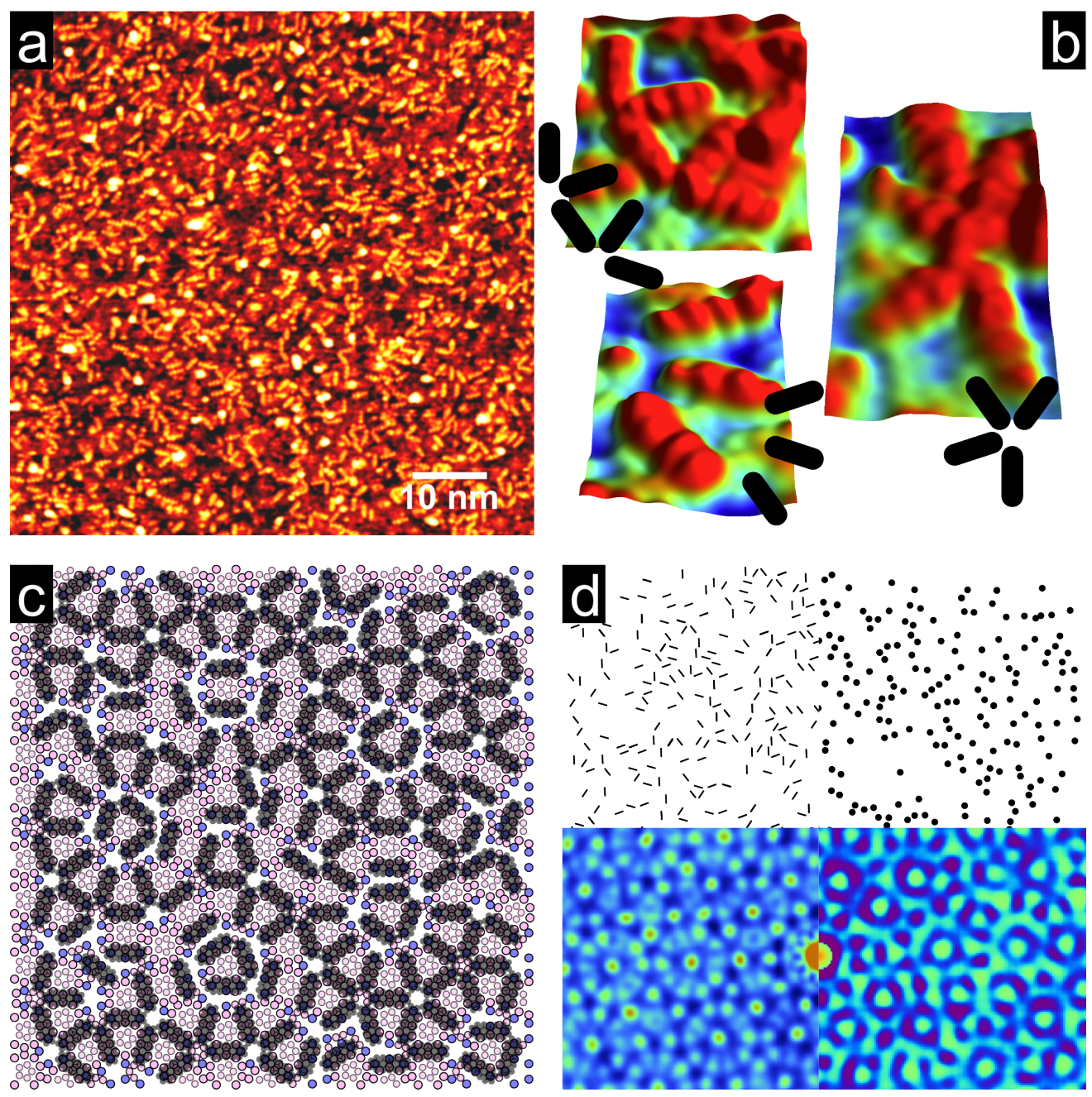

Figure 2: Quasicrystalline Pn layer on icosahedral Ag-In-Yb.(a) $75 \mathrm{~nm} \times 75 \mathrm{~nm}$ STM image of Pn molecules adsorbed atop Ag-In- $\mathrm{Yb}\left(V_{\text {gap }}=-2 \mathrm{~V}, I_{T}=0.2 \mathrm{nA}\right)$. (b) Details of highresolution scans over the areas indicated in (a), showing molecules arranged in motifs with pentagonal symmetry with four local density-of-states (LDOS) maxima per molecule. The relevant motifs are shown in black to assist the reader. (c) A $20 \mathrm{~nm}$ square of the Ag-In-Yb surface model saturated with Pn molecules. Pink atoms are Ag or In, purple atoms are Yb. (d) Molecular positions extracted and plotted with (left) and without (right) orientation information. The autocorrelation functions shown below indicate the increased quality of the quasicrystalline ordering when the orientation of the Pn molecules is included.

fold (LUMO) or five-fold (HOMO) nature of the frontier orbitals is clearly visible. ${ }^{31}$

Pn adopts quasiperiodic sites on the surface, shown in Figure 2(a) and in detail in Figure 2(b). Pn molecules appear as four-lobed features, as shown in Figure 2(c). This is not observed in any other STM studies of Pn, and, since adsorption at RT without subsequent annealing implies that it is unlikely that the molecules undergo 

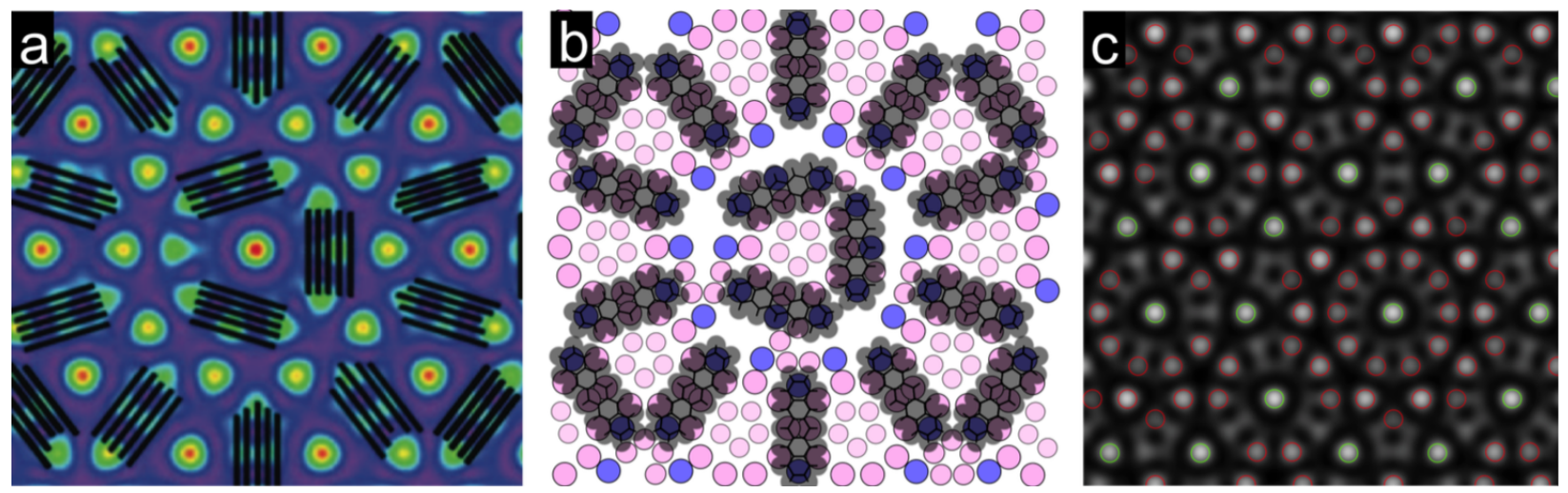

Figure 3: Comparison to computational study. (a) The distribution of spherocylindrical rods in a 2-dimensional quasicrystalline potential generated by the simulated interference of five laser beams. Reproduced from reference. ${ }^{36}$ (b) A detail from the model of the saturated layer shown in figure 2(c) showing the exact correspondence of motifs. (c) A larger image of the potential surface shown in (a) with Yb atoms (red) and Ag-In-Yb cluster centers (green) superimposed. Reproduced from reference. ${ }^{37}$

any structural modification, indicates that there is some electronic disruption of the molecule. The two-fold symmetry of the molecules indicates that this disruption occurs symmetrically or nearly symmetrically within the molecule; therefore, we can infer that bonds are formed either at the center, or at locations equidistant about the center, of the molecule with some feature or equivalent features of the substrate.

The motifs shown in Figure 2(b) provide a way to determine the adsorption sites for the Pn molecules. The structural quality of the layer indicates that there are few competing possibilities for adsorption sites. If one atomic arrangement on the quasicrystalline surface is found in similar arrangements to the quasicrystalline Pn layer, then the distribution of such sites is likely to constitute the adsorption site network. We find only one arrangement that can both provide an adsorption site network which produces the observed motifs and is symmetrical enough to provide a kind of interaction that might result in the four-fold modification of the Pn LUMO. Several other nucleation site networks were considered and each alternative failed to reproduce some motif that was observed in the data. These are described in Supplemental Information in Figure S4.

A model with one permutation of a saturated layer consisting of molecules in such 
sites, characterised by two $\mathrm{Yb}$ atoms at a separation consistent with the endmost aromatic rings of the $\mathrm{Pn}$ molecules, is shown in Figure 2(c). As for the $\mathrm{C}_{60} / \mathrm{Al}-\mathrm{Cu}-\mathrm{Fe}$ system, clear ten-fold symmetry is present in the molecular layer. As before, we demonstrate this by extracting the positions of the molecules in STM images and calculating the autocorrelation function of the resultant binary map. In this system we also have the opportunity to investigate the orientations as well as the positions of the molecules. When this information is included in the autocorrelation, the quality improves, as shown in Figure 2(d).

This network exhibits quasi-degeneracy similar to $\mathrm{C}_{60} / \mathrm{Al}-\mathrm{Cu}-\mathrm{Fe}$. However, there is no coexisting non-degenerate network. In addition, the nucleation site is not based on the centers of the rhombic triacontahedral clusters that make up the icosahedral $\mathrm{Ag}-\mathrm{In}-\mathrm{Yb}$ lattice, but rather is determined solely by an interaction that occurs with ten-fold symmetry around all RTH clusters. The two-fold symmetry in the distortion of the electron cloud around the Pn molecules combined with a comparison of possible nucleation sites given in Supplemental Information S4 suggests that this interaction is the formation of two bonds, each between a terminal Pn benzene ring and a substrate $\mathrm{Yb}$ atom. Most $\mathrm{Yb}$ atoms occur in decagons around surface-bisected RTH clusters.

In Figure 3 we compare the adsorption site thus determined with a relevant preexisting study by Kählitz et al. ${ }^{36}$ In this study, rods consisting of cylinders terminated in half-spheres of the same radius as the rod, were allowed to diffuse on a quasicrystalline potential generated by the simulated interference of five laser beams arranged in fivefold symmetry. Clusters of short rods were shown to assemble in the manner shown in Figure 3, with each cluster of 5 rods occupying a space spanning three minima in the potential energy surface. In terms of the relationship between the characteristic sizes of the rods and the potential energy surface, there is a good match between Pn molecules and the 5 -rod clusters described in the reference. ${ }^{36}$ The simulated potential, a larger example of which is reproduced in Figure 3(c), shows a large degree of correspondence with the Ag-In-Yb surface, which is represented as open circles denoting the locations of surface $\mathrm{Yb}$ atoms (red) and cluster centers (green). This comparison indicates that, 
despite the complicated nature of the Ag-In-Yb surface, the potential energy surface for the formation of this overlayer is relatively simple.

The existence of a network of well-separated but well-defined atomic locations is a quality unlikely to be unique to quasicrystals. Complex metallic alloys (CMAs), including quasicrystal approximants, are periodic materials with large unit cells. Approximants exist in the phase diagrams of both $\mathrm{Al}-\mathrm{Cu}-\mathrm{Fe}$ and $\mathrm{Ag}-\mathrm{In}-\mathrm{Yb}$. Periodic quasi-degenerate systems would be of more technological interest than aperiodic systems, due to the greater predictability of sites in the quasi-degenerate lattice. Switching of molecules between adjacent exclusive lattice sites could provide low-energy nanoscale data storage, in a similar vein to the 'molecular abacus' ${ }^{38}$ and $\mathrm{C}_{60}$ polymerization memory. ${ }^{39}$

Another aspect relates to the demonstration of quasicrystalline ordering on a larger length scale than previously encountered. While these studies do not constitute quasicrystalline self-assembly, they point to the possibility of using templated molecular systems to produce larger scale systems with the isotropic photonic band gap demonstrated for metamaterial quasicrystals.

The question of the conflict between coverage and order also arises in these studies. The sticking rate for $\mathrm{C}_{60}$ on $\mathrm{Al}-\mathrm{Cu}-\mathrm{Fe}$ is greatly reduced at $773 \mathrm{~K}$. However, this elevated temperature is required to permit the molecules to diffuse to preferential adsorption sites and to inhibit sticking at disordered sites. Therefore, it seems that at saturation level for the ordered layer, no further disordered $\mathrm{C}_{60}$ will stick. In contrast, for Pn on $\mathrm{Ag}-\mathrm{In}-\mathrm{Yb}$, the molecules adopt ordered positions without annealing. In this case, the conflict between coverage and ordering is very apparent and is covered in more detail in Supplemental Information in Figure S5.

In conclusion, we present STM images demonstrating the first molecular systems that exhibit two-dimensional hierarchical quasicrystalline growth. We demonstrate spherical and rod-shaped molecules in quasicrystalline lattices, thus exploring molecular orientation as well as location, and finding that orientation offers an additional degree of freedom with which to exhibit quasicrystalline ordering. The motifs we observe are 
remarkably similar to calculations of finite-width rods in a quasicrystalline potential. The quasi-degenerate adsorption site network is demonstrated in each case. Finally, we point out the possibility of using templated molecular adsorption to produce metamaterials with quasicrystalline properties, such as an isotropic band gap, on a chosen length scale.

\section{Acknowledgments}

The UK EPSRC is thanked for funding under grant number EP/D05253X/1. We thank T. A. Lograsso and A. R. Ross of Ames Laboratory for provision of the Al-Cu-Fe sample. We thank G. Teobaldi and H. Stark for interesting discussions and correspondence. JAS acknowledges support through the UK Science \& Innovation Network and Department for Business, Innovation, and Skills. HRS is thankful to EPSRC for funding under Grant no EP/D071828/1.

\section{Methods}

The quasicrystal samples were prepared using the standard sputter and anneal pro-

cedures described elsewhere. ${ }^{26,32}$ The experiments were performed in several Omicron RT-STM 1 systems and an Omicron VT-STM system. The molecules were evaporated from separate homemade sources, each consisting of a Pyrex tube wrapped with a Ta filament with a K-type thermocouple for temperature regulation. Pn molecules were evaporated at 390 and $\mathrm{C}_{60}$ molecules were evaporated at $500 \mathrm{~K}$. Substrate temperatures during deposition were $300 \mathrm{~K}$ for $\mathrm{Pn} / \mathrm{Ag}$-In-Yb and $773 \mathrm{~K}$ to $973 \mathrm{~K}$ for $\mathrm{C}_{60} / \mathrm{Al}-\mathrm{Cu}-\mathrm{Fe}$. High sample temperatures were monitored with a Minolta LAND Cyclops 341 optical pyrometer with emissivity set to 0.35 . Following deposition, the molecule/quasicrystal system was moved to the STM and studied at room temperature. 


\section{References}

(1) Shechtman, D.; Blech, I.; Gratias, D. Phys. Rev. Lett. 1984, 53, 1951.

(2) Penrose, R. Bull. Inst. Math. Appl. 1974, 10, 266-271.

(3) Tsai, A.-P.; Guo, J. Q.; Abe, E.; Takakura, H.; Sato, T. J. Nature 2000, 408, $537-538$.

(4) Franke, K. J.; Sharma, H. R.; Theis, W.; Gille, P.; Ebert, P.; Rieder, K. H. Phys. Rev. Lett. 2002, 89, 156104.

(5) Smerdon, J. A.; Parle, J. K.; Wearing, L. H.; Lograsso, T. A.; Ross, A. R.; McGrath, R. Phys. Rev. B 2008, 78, 075407.

(6) Ledieu, J.; Krajčí, M.; Hafner, J.; Leung, L.; Wearing, L. H.; McGrath, R.; Lograsso, T. A.; Wu, D.; Fournée, V. Phys. Rev. B 2009, 79, 165430.

(7) Sharma, H. R.; Shimoda, M.; Ross, A. R.; Lograsso, T. A. Phys. Rev. B 2005, 72, 045428.

(8) Ledieu, J.; Unsworth, P.; Lograsso, T. A.; Ross, A. R.; McGrath, R. Phys. Rev. B 2006, 73, 012204.

(9) Cai, T.; Ledieu, J.; McGrath, R.; Fournée, V.; Lograsso, T. A.; Ross, A. R.; Thiel, P. Surf. Sci. 2003, 526, 115-120.

(10) Wearing, L. H.; Smerdon, J. A.; Leung, L.; Dhesi, S. S.; Ledieu, J.; Bencok, P.; Fisher, I.; Jenks, C. J.; McGrath, R. J. Phys.: Condens. Matter 2007, 20, 015005.

(11) Ledieu, J.; Hoeft, J. T.; Reid, D. E.; Smerdon, J. A.; Diehl, R. D.; Lograsso, T. A.; Ross, A. R.; McGrath, R. Phys. Rev. Lett. 2004, 92, 135507.

(12) Smerdon, J. A.; Ledieu, J.; Hoeft, J. T.; Reid, D. E.; Wearing, L. H.; Diehl, R. D.; Lograsso, T. A.; Ross, A. R.; McGrath, R. Phil. Mag. 2006, 86, 841-847. 
(13) Zeng, X.; Ungar, G.; Liu, Y.; Percec, V.; Dulcey, A. E.; Hobbs, J. K. Nature 2004, 428, 157-160.

(14) Talapin, D. V.; Shevchenko, E. V.; Bodnarchuk, M. I.; Ye, X. Nature 2009, 461, 964-967.

(15) Mikhael, J.; Roth, J.; Helden, L.; Bechinger, C. Nature 2008, 454, 501-504.

(16) Förster, S.; Meinel, K.; Hammer, R.; Trautmann, M.; Widdra, W. Nature 2013, 502, 215-218.

(17) Ungar, G.; Zeng, X. Soft Matter 2005, 1, 95-106.

(18) Blunt, M. O.; Russell, J. C.; Giménez-López, M. C.; Garrahan, J. P.; Lin, X.; Schröder, M.; Champness, N. R.; Beton, P. H. Science 2008, 322, 1077.

(19) Vedmedenko, E. Y.; Oepen, H. P.; Kirschner, J. Phys. Rev. Lett. 2003, 90, 137203.

(20) Ledieu, J.; Muryn, C. A.; Thornton, G.; Diehl, R. D.; Lograsso, T. A.; Delaney, D. W.; McGrath, R. Surf. Sci. 2001, 472, 89-96.

(21) Smerdon, J. A.; Parle, J. K.; Wearing, L. H.; Leung, L.; Lograsso, T. A.; Ross, A. R.; McGrath, R. J. Phys.: Conf. Ser. 2010, 226, 012006.

(22) Young, K. M.; Smerdon, J. A.; Sharma, H. R.; Lahti, M.; Pussi, K.; McGrath, R. Phys. Rev. B 2013, 87, 085407.

(23) Smerdon, J. A.; Leung, L.; Parle, J. K.; Jenks, C. J.; McGrath, R.; Fournée, V.; Ledieu, J. Surface Science 2008, 602, 2496-2501.

(24) Boudard, M.; Boissieu, M. d.; Janot, C.; Dubois, J.-M.; Dong, C. Philosophical Magazine Letters 1991, 64, 197-206.

(25) Yamamoto, A.; Takakura, H.; Tsai, A. P. Phys. Rev. B 2003, 68.

(26) Sharma, H. R.; Fournée, V.; Shimoda, M.; Ross, A. R.; Lograsso, T. A.; Tsai, A. P.; Yamamoto, A. Phys. Rev. Lett. 2004, 93, 165502. 
(27) Ledieu, J.; McGrath, R. J. Phys.: Condens. Matter 2003, 15, S3113.

(28) Unal, B.; Fournée, V.; Schnitzenbaumer, K. J.; Ghosh, C.; Jenks, C. J.; Ross, A. R.; Lograsso, T. A.; Evans, J. W.; Thiel, P. A. Phys. Rev. B 2007, 75, 064205 .

(29) Ünal, B.; Jenks, C. J.; Thiel, P. A. J. Phys.: Condens. Matt. 2009, 21, 055009.

(30) Cai, T.; Shi, F.; Shen, Z.; Gierer, M.; Goldman, A. I.; Kramer, M. J.; Jenks, C. J.; Lograsso, T. A.; Delaney, D. W.; Thiel, P. A.; Van Hove, M. A. Surf. Sci. 2001, $495,19-34$.

(31) Smerdon, J. A.; Bode, M.; Guisinger, N. P.; Guest, J. R. Phys. Rev. B 2011, 84, 165436.

(32) Sharma, H. R.; Shimoda, M.; Sagisaka, K.; Takakura, H.; Smerdon, J. A.; Nugent, P. J.; McGrath, R. Phys. Rev. B 2009, 80, 121401.

(33) Lagoute, J.; Kanisawa, K.; Fölsch, S. Phys. Rev. B 2004, 70, 245415.

(34) Dougherty, D. B.; Jin, W.; Cullen, W. G.; Reutt-Robey, J. E.; Robey, S. J. Phys. Chem. C 2008, 112, 20334-20339.

(35) Repp, J.; Meyer, G.; Stojković, S. M.; Gourdon, A.; Joachim, C. Phys. Rev. Lett. 2005, 94, 026803.

(36) Kählitz, P.; Schön, M.; Stark, H. J. Chem. Phys. 2012, 137, 224705-224705-11.

(37) Schmiedeberg, M.; Roth, J.; Stark, H. Eur. Phys. J. E 2007, 24, 367-377.

(38) Cuberes, M. T.; Schittler, R. R.; Gimzewski, J. K. Appl. Phys. Lett. 1996, 69, 3016-3018.

(39) Okawa, Y.; Aono, M. Nature 2001, 409, 683-684. 